Published in final edited form as:

J Med Chem. 2012 August 9; 55(15): 6985. doi:10.1021/jm300948p.

\title{
Correction to first total synthesis of protoapigenone and its analogues as potent cytotoxic agents.
}

Manuscript number of paper to be corrected: jm070363a

Reference citation of paper to be corrected: J. Med. Chem. 2007, 50, 3921-3927

An-Shen Lin, Kyoko Nakagawa-Goto, Fang-Rong Chang, Donglei Yu, Susan L. MorrisNatschke, Chin-Chung Wu, Shu-Li Chen, Yang-Chang Wu,* and Kuo-Hsiung Lee*: First Total Synthesis of Protoapigenone and Its Analogues as Potent Cytotoxic Agents

Page 3922. The abbreviations of benzyl protecting groups in scheme 1 were mistakenly drawn. They should be "Bn" instead of "Bz". The correct scheme is shown below.

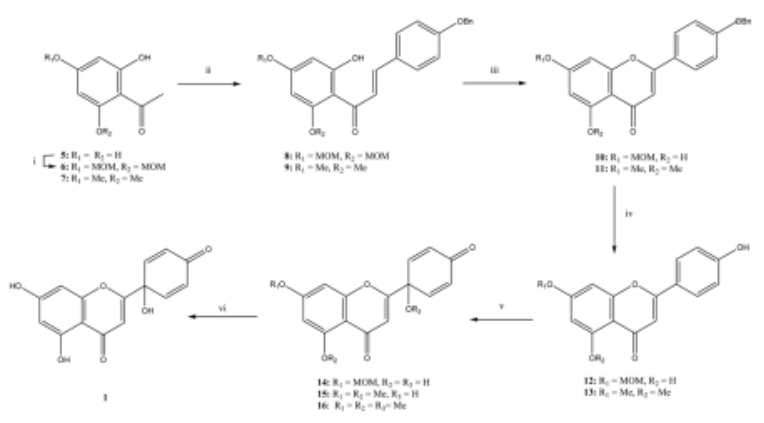

